\title{
Clinical value of color Doppler ultrasound combined with serum CA153, CEA and TSGF detection in the diagnosis of breast cancer
}

\author{
XIUQIN SONG ${ }^{*}$, BO LIANG ${ }^{2 *}$, CHENGHONG WANG $^{3}$ and SHAN SHI ${ }^{4}$ \\ ${ }^{1}$ Department of Ultrasound, Yantaishan Hospital; ${ }^{2}$ Department of Ultrasound, Yantai Hospital of Traditional Chinese Medicine; \\ ${ }^{3}$ Department of Clinical Laboratory, Yantaishan Hospital, Yantai, Shandong 264000; ${ }^{4}$ Department of Ultrasound, \\ Shandong Medical Imaging Research Institute, Jinan, Shandong 250021, P.R. China
}

Received December 3, 2019; Accepted May 12, 2020

DOI: $10.3892 /$ etm. 2020.8868

\begin{abstract}
In the present retrospective study, the clinical value of color Doppler ultrasound combined with the detection of the serum tumor markers carbohydrate antigen 153 (CA153), carcinoembryonic antigen (CEA) and tumor specific growth factor (TSGF) as diagnostic tools for breast cancer was investigated. A total of 103 patients with breast cancer and 50 patients with benign breast lesions were enrolled in the study. All patients were examined by color Doppler ultrasonography. Electrochemiluminescence was used to measure the expression levels of CA153 and CEA, and chemical colorimetric assay for the measurement of TSGF expression. The differences in mass boundary, morphology, internal echo, calcification, peak blood flow velocity $\left(\mathrm{V}_{\max }\right)$, resistance index (RI), pulsatility index (PI) and blood flow signal classification between the two groups were statistically significant $(\mathrm{P}<0.05)$. The expression levels of CA153, CEA and TSGF in the serum of patients in the breast cancer group were significantly higher than those in the benign lesion group $(\mathrm{P}<0.01)$. In the breast cancer group, CA153, CEA and TSGF expression levels were significantly higher in patients with high-stage cancer and recurrence, compared with the patients with low-stage cancer and no recurrence. The sensitivity, accuracy and negative predictive value of the combined detection were significantly improved compared with those of the single tests $(\mathrm{P}<0.01)$. Color Doppler ultrasound combined with the detection of CA153, CEA and TSGF levels in the serum of patients can be used as an effective tool that can improve the accuracy of breast cancer diagnosis leading to early diagnosis and clinical intervention.
\end{abstract}

Correspondence to: Dr Shan Shi, Department of Ultrasound, Shandong Medical Imaging Research Institute, 324 Jingwu Road, Jinan, Shandong 250021, P.R. China

E-mail:10b8i9@163.com

*Contributed equally

Key words: breast cancer, color doppler ultrasound, tumor markers, joint examination, diagnosis

\section{Introduction}

Breast cancer is one of the most common malignant tumors among women. In recent years, the rising incidence of breast cancer which has exceeded that of cervical cancer has attracted the attention of medical societies (1). Due to the hidden nature of breast cancer symptoms at early stage, most patients come to the hospital at the middle or late stage of breast cancer, missing the best treatment opportunity. Early diagnosis and treatment are essential for better prognosis (2). The preferred diagnostic method for breast cancer is color Doppler ultrasonography. Ultrasound can show the internal structure and blood flow in breast masses, and can reveal the shape, boundary and activity of the lesions. In addition, ultrasound can distinguish solid and cystic masses (3). However, its application has certain limitations as the distinction between small lesions and some benign and malignant masses is very difficult. In recent years, with the development of molecular biology techniques, more tumor molecular markers related to breast cancer have been discovered. The evaluation of the expression levels of these markers in the serum of patients can provide precious information related to the occurrence, invasion and metastasis of breast cancer (4). Among these markers, carbohydrate antigen 153 (CA153), carcinoembryonic antigen (CEA) and tumor specific growth factor (TSGF) are closely related to breast cancer occurrence and their detection could be used as an auxiliary examination method for breast cancer diagnosis. Although the combined diagnostic method could improve the accuracy of breast cancer diagnosis, no standard diagnostic method has been developed. In the present study, color Doppler ultrasound combined with the detection of serum markers CA153, CEA and TSGF was used in the diagnosis of breast cancer, aiming to provide future reference for the early diagnosis of breast cancer.

\section{Patients and methods}

Clinical data. From January 2017 to June 2018, a total of 103 patients with breast cancer admitted to Yantaishan Hospital (Yantai, China) were enrolled in the study and served as the breast cancer group. All cases were confirmed by pathological and histological examinations. Patients were all females, 35-79 years of age, with an average age of $56.32 \pm 12.47$ years. Regarding tumor location, there 
were 63 patients with tumors in the left breast, 37 patients with tumors in the right breast and 3 patients with bilateral breast cancer. According to the tumor-node-metastasis classification standard of breast cancer published by the Union for International Cancer Control (5), there were 26 cases at stage I, 35 cases at stage II, 30 cases at stage III and 12 cases at stage IV. The histopathological types of cancer included carcinoma in situ, invasive ductal carcinoma, invasive lobular carcinoma and medullary carcinoma. Furthermore, 50 patients with benign breast lesions were selected as the benign lesion group. All patients in the benign lesion group were females, 33-80 years of age, with an average age of $55.32 \pm 13.01$ years. Canceration was excluded by pathological histology, including breast fibroadenoma, breast papilloma, breast hyperplasia and breast cyst. There was no statistically significant difference between the two groups in terms of sex, age and other general characteristics $(\mathrm{P}>0.05)$.

Inclusion criteria for breast cancer patients: i) All patients met the criteria of the breast cancer staging system (6) as confirmed by pathological examinations; ii) patients had not been diagnosed before with breast cancer and had not received radiotherapy, chemotherapy or other endocrine therapy before treatment; iii) patients underwent color Doppler ultrasound and serum CA153, CEA and TSGF examinations. Exclusion criteria: Patients with related tumor diseases or liver and kidney dysfunction. The study was approved by the Ethics Committee of Yantaishan Hospital. Patients who participated in the study had complete clinical data. Signed written informed consents were obtained from the patients and/or guardians.

Color Doppler ultrasound examination. Color Doppler ultrasound diagnosis was based on the Breast Imaging-Reporting and Data System developed by the American College of Radiology for the evaluation of breast cancer imaging (7). Philips iU22 (Philips Healthcare) was the diagnostic instrument used and the probe frequency was 5-12 MHz. Patients were instructed to lie in supine position, lift their arms and place their hands behind the head in order to fully expose the bilateral breasts, supraclavicular fossa and bilateral axillary regions. A probe was used to scan radially along the areola with the nipple as the center. At the same time, the supraclavicular fossa and bilateral armpits were scanned. Firstly, the location, shape, size, boundary, internal echo, calcification, correlation with surrounding tissues, axillary lymph node metastasis and other acoustic images were observed. Next, the morphology and distribution of blood flow signals inside and around the focus were examined and hemodynamic parameters were measured (8).

\section{Serum tumor markers}

Specimen collection and pretreatment. A total of $4 \mathrm{ml}$ of cubital venous blood were collected from all patients in both groups, on an empty stomach at 7 a.m., before treatment and 3 months after treatment. After autocoagulation at $25^{\circ} \mathrm{C}$, the blood samples were centrifuged at 2,264 $\mathrm{x}$ g for $20 \mathrm{~min}$ at $25^{\circ} \mathrm{C}$ and the upper serum was collected for examination. Lipids and hemolysis were excluded from all specimens.

Determination of serum CA153 and CEA expression levels. An electrochemiluminescence immunoassay was used for the detection of CA153 and CEA expression levels, following the principles of double antibody sandwich. The instrument used was Roche Elecsys 2010 with CA153 (cat. no. 03045838122) and CEA (cat. no. 11731629322) diagnostic reagents provided by Roche Applied Science. Step 1: Each sample was firstly diluted (1:10) with Elecsys universal diluent. CA153 and CEA antigens formed immune sandwich complexes with biotinylated anti-CA153 and CEA monoclonal antibodies and ruthenium-labeled anti-CA153 and CEA antibodies in the reagent, respectively. Step 2: Streptavidin-coated particles were automatically added by Elecsys 2010, allowing the complexes to bind to the particles through the reaction of biotin with streptavidin. Step 3: The reaction mixture was sucked into the cells and the particles were attracted to the electrode by a magnet. The free substance was washed away by a cleaning solution and the immune complex generated chemiluminescence after voltage was applied to the electrodes. Chemiluminescence activity was measured by a photomultiplier tube (Roche Applied Science). Step 4: The standard curve was calibrated by the instrument via a 2-point calibration. The measurement results were automatically detected from the standard curve. When the sample exceeded the linear range, the instrument automatically diluted the sample using a universal diluent. The whole process was fully automatic and the actual serum dosage in each experiment was $20 \mu 1$.

TSGF detection. Chemical colorimetric assay was performed using a Hitachi 7600-110E (Hitachi, Ltd.) with TSGF kit (cat. no. A0229) provided by Newland Biotech. All operation steps were carried out following strictly the manufacturer's instructions. Step 1: TSGF reagents, calibrators and quality control materials were positioned as designated. Step 2: The samples were placed at the specified position to perform the test. After the completion of the test, the results were obtained based on the standard curve.

Result determination. With pathological diagnosis as the gold standard, the cases in the breast cancer group with consistent results of ultrasound and pathological diagnosis were determined as true positives. The cases with inconsistent results (misdiagnosis, missed diagnosis and uncertainty) were determined as false negatives. In the benign lesion group, the cases with consistent results of ultrasound and pathological diagnosis were determined as true negatives, whereas the cases with inconsistent results (misdiagnosis, uncertainty) were determined as false positive. When one or more items in the combined detection (parallel test) group were positive, the case was determined as positive. If all items were negative, the case was determined as negative.

Statistical analysis. SPSS 19.0 software (IBM Corp.) was used for the statistical analysis of the data. Measured data were expressed as the mean \pm standard deviation (mean \pm SD) and the comparison of the mean values between groups was made using independent samples t-test. The comparison of the counting data was made using $\chi^{2}$ test. Receiver operating characteristics (ROC) curve analysis of CA153, CEA and TSGF was also performed and the optimal cut-off values for CA153, CEA and TSGF in diagnosing breast cancer were 
Table I. Comparison of color Doppler ultrasonography features between patients with breast cancer and patients with benign lesions $[\mathrm{n}(\%)]$.

\begin{tabular}{|c|c|c|c|c|c|c|c|c|}
\hline \multirow[b]{2}{*}{ Group } & \multicolumn{2}{|c|}{ Boundary } & \multicolumn{2}{|c|}{ Internal echo } & \multicolumn{2}{|c|}{ Morphology } & \multicolumn{2}{|c|}{ Calcification } \\
\hline & Clear & Unclear & Homogeneous & Inhomogeneous & Regular & Irregular & No & Yes \\
\hline $\begin{array}{l}\text { Breast cancer } \\
\text { group }(n=103)\end{array}$ & $18(17.48)^{\mathrm{a}}$ & $85(82.52)^{\mathrm{a}}$ & $25(24.27)^{\mathrm{a}}$ & $78(75.73)^{\mathrm{a}}$ & $16(15.53)^{\mathrm{a}}$ & $87(84.47)^{\mathrm{a}}$ & $14(13.59)^{\mathrm{a}}$ & $89(86.41)^{a}$ \\
\hline $\begin{array}{l}\text { Benign lesion } \\
\text { group }(n=50)\end{array}$ & $40(80.00)$ & $10(20.00)$ & $35(70.00)$ & $15(30.00)$ & $38(76.00)$ & $12(24.00)$ & $41(82.00)$ & $9(18.00)$ \\
\hline
\end{tabular}

${ }^{\text {aP }}<0.05$, compared with the benign lesion group.

Table II. Comparison of color Doppler ultrasound blood flow features between patients with breast cancer and patients with benign lesions [mean $\pm \mathrm{SD}, \mathrm{n}(\%)]$.

Blood flow signal classification

\begin{tabular}{lccccccc}
\cline { 5 - 7 } Group & $\mathrm{V}_{\max }(\mathrm{cm} / \mathrm{sec})$ & $\mathrm{RI}$ & PI & 0 & $\mathrm{I}$ & II & III \\
\hline Breast cancer group $(\mathrm{n}=103)$ & $25.63 \pm 3.26^{\mathrm{a}}$ & $0.82 \pm 0.05^{\mathrm{a}}$ & $1.65 \pm 0.31^{\mathrm{a}}$ & $6(5.83)^{\mathrm{a}}$ & $15(14.56)^{\mathrm{a}}$ & $27(26.21)^{\mathrm{a}}$ & $55(53.40)^{\mathrm{a}}$ \\
Benign lesion group $(\mathrm{n}=50)$ & $13.69 \pm 2.01$ & $0.61 \pm 0.03$ & $1.02 \pm 0.21$ & $25(50.00)$ & $16(32.00)$ & $8(16.00)$ & $1(2.00)$ \\
\hline
\end{tabular}

${ }^{\mathrm{a}} \mathrm{P}<0.05$, compared with the benign lesion group. Vmax, peak blood flow velocity; RI, resistance index; PI, pulsatility index.

determined according to the maximum Youden index. $\mathrm{P}<0.05$ was considered to indicate a statistically significant difference.

\section{Results}

Results of color Doppler ultrasonography in the breast cancer and the benign lesion group. A total of 103 cases of breast cancer were examined by color Doppler ultrasound. The pathological results were consistent with the ultrasound results in 80 cases (confirmed in 80 cases) and inconsistent in 23 cases (misdiagnosed in 8 cases, missed in 3 cases, uncertain in 12 cases). In the benign lesion group $(n=50)$, the pathological results were consistent with the ultrasound results in 42 cases (confirmed in 42 cases) and inconsistent in 8 cases (misdiagnosed in 3 cases and uncertain in 5 cases).

Color Doppler ultrasound detection showed that there were statistically significant differences between the two groups in terms of tumor boundary, morphology, internal echo, calcification, peak blood flow velocity $\left(\mathrm{V}_{\max }\right)$, resistance index $(\mathrm{RI})$, pulsatility index (PI) and blood flow classification (0, I, II, III) ( $\mathrm{P}<0.05$; Tables I and II). Color Doppler ultrasound images of breast cancer showed the unclear boundaries of the tumors, the tumors' irregular shape, inhomogeneous internal echo, calcification, as well as the increase of $\mathrm{V}_{\max }$, RI and PI of blood flow. The internal blood flow of the tumors was also shown to be rich. Representative ultrasonic images are shown in Fig. 1.

Comparison of serum tumor markers between the breast cancer and the benign lesion group. The serum levels of tumor markers CA153, CEA and TSGF in the patients with breast cancer were significantly higher than those in the patients with

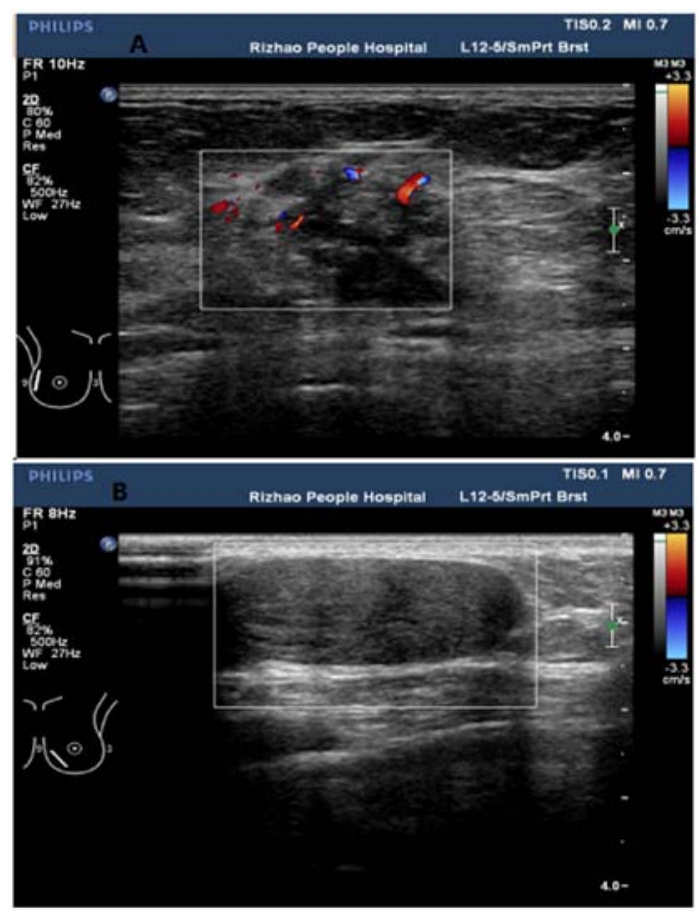

Figure 1. Representative color Doppler ultrasound images. (A) Color Doppler ultrasound image of a malignant tumor. The boundary of the tumor was unclear, the shape was irregular, with crab-like infiltration and the internal echo was low and inhomogeneous. In addition, spot-like strong echo was observed. The echo behind the tumor was attenuated and a high-resistance and high-speed blood flow signal was observed around and inside the tumor. Resistance index, 0.73. (B) Color Doppler ultrasound image of a benign lesion. The shape of the tumor was elliptical, the boundary was clear, and the internal echo was low and homogeneous. In addition, side acoustic shadows could be observed on both sides of the tumor and there was no echo change behind the tumor. According to color Doppler flow imaging, there was no obvious blood flow signal in the tumor. 
Table III. Comparison of the expression levels of serum tumor markers between patients with breast cancer and patients with benign lesions (mean $\pm \mathrm{SD}$ ).

\begin{tabular}{llcr}
\hline Group & CAl53 $(\mathrm{U} / \mathrm{ml})$ & CEA $(\mathrm{ng} / \mathrm{ml})$ & TSGF $(\mathrm{U} / \mathrm{ml})$ \\
\hline Breast cancer group $(\mathrm{n}=103)$ & $72.61 \pm 23.40^{\mathrm{a}}$ & $36.24 \pm 16.25^{\mathrm{a}}$ & $157.69 \pm 46.72^{\mathrm{a}}$ \\
Benign lesion group $(\mathrm{n}=50)$ & $14.67 \pm 8.76$ & $2.36 \pm 0.82$ & $50.24 \pm 15.61$ \\
\hline
\end{tabular}

${ }^{a} \mathrm{P}<0.01$, compared with the benign lesion group. CA153, carbohydrate antigen 153 ; CEA, carcinoembryonic antigen; TSGF, tumor specific growth factor.

Table IV. Comparison of CA153, CEA and TSGF serum levels in patients with different clinical features in the breast cancer group $($ mean $\pm \mathrm{SD})$.

\begin{tabular}{|c|c|c|c|c|}
\hline Group & Cases & CAl53 (U/ml) & $\mathrm{CEA}(\mathrm{ng} / \mathrm{ml})$ & TSGF (U/ml) \\
\hline \multicolumn{5}{|c|}{ Clinical stage } \\
\hline $\mathrm{I}+\mathrm{II}$ & 61 & $46.92 \pm 12.29$ & $23.58 \pm 10.12$ & $120.53 \pm 36.12$ \\
\hline III+IV & 42 & $110.83 \pm 36.12^{\mathrm{a}}$ & $55.64 \pm 23.14^{\mathrm{a}}$ & $212.09 \pm 70.21^{\mathrm{a}}$ \\
\hline \multicolumn{5}{|c|}{ Distant metastasis } \\
\hline No & 91 & $56.83 \pm 15.64$ & $26.54 \pm 11.15$ & $128.72 \pm 40.15$ \\
\hline Yes & 12 & $196.17 \pm 67.23^{\mathrm{a}}$ & $110.48 \pm 36.57^{\mathrm{a}}$ & $378.33 \pm 97.26^{\mathrm{a}}$ \\
\hline \multicolumn{5}{|c|}{ Recurrence } \\
\hline No & 82 & $19.37 \pm 9.21$ & $5.62 \pm 2.73$ & $96.31 \pm 22.14$ \\
\hline Yes & 21 & $96.49 \pm 43.27^{\mathrm{a}}$ & $47.37 \pm 18.87^{\mathrm{a}}$ & $203.39 \pm 66.42^{\mathrm{a}}$ \\
\hline \multicolumn{5}{|c|}{ Treatment } \\
\hline Before & 103 & $72.61 \pm 23.40$ & $36.24 \pm 16.25$ & $157.69 \pm 46.72$ \\
\hline After & 103 & $34.21 \pm 20.15^{\mathrm{a}}$ & $13.52 \pm 6.82^{\mathrm{a}}$ & $107.82 \pm 32.97^{\mathrm{a}}$ \\
\hline
\end{tabular}

${ }^{\mathrm{a}} \mathrm{P}<0.01$, for intragroup comparisons. CA153, carbohydrate antigen 153; CEA, carcinoembryonic antigen; TSGF, tumor specific growth factor.

Table V. Association of color Doppler ultrasound, CA153, CEA, TSGF single and combined detection results with pathological diagnosis $(n)$.

Pathological

diagnosis

\begin{tabular}{llcr}
\cline { 3 - 3 } Detection & Detection result & Positive & Negative \\
\hline Ultrasound & Positive & 80 & 8 \\
& Negative & 23 & 42 \\
CA153 & Positive & 66 & 4 \\
CEA & Negative & 37 & 46 \\
TSGF & Positive & 64 & 5 \\
& Negative & 39 & 45 \\
Combined detection & Positive & 68 & 5 \\
& Negative & 35 & 45 \\
& Positive & 98 & 10 \\
& Negative & 5 & 40
\end{tabular}

CA153, carbohydrate antigen 153; CEA, carcinoembryonic antigen; TSGF, tumor specific growth factor. benign lesions $(\mathrm{P}<0.01$; Table III). The expression levels of these tumor markers in patients with high-stage cancer (III, IV), recurrence and metastasis of breast cancer were significantly higher than those with low-stage cancer (I, II), no recurrence and no metastasis. The expression levels of CA153, CEA and TSGF after breast cancer treatment were significantly lower than those before treatment $(\mathrm{P}<0.01$; Table IV).

Comparison of the clinical value of color Doppler ultrasound, CA153, CEA, TSGF single and combined detection in the diagnosis of breast cancer. ROC curve analysis revealed that the area under the curve (AUC) of CA153, CEA and TSGF in the diagnosis of breast cancer was $0.812,0.807$ and 0.843 , respectively, and the maximum Youden index was $0.568,0.521$ and 0.560 , respectively. The best cut-off values were $25.00 \mathrm{U} / \mathrm{ml}$ for CA153, $3.40 \mathrm{ng} / \mathrm{ml}$ for CEA and $70.00 \mathrm{U} / \mathrm{ml}$ for TSGF. The consistency of the results of ultrasonography, CA153, CEA, TSGF single and combined detection with the results of the pathological diagnosis is presented in Table V. The sensitivity, accuracy and negative predictive value of color Doppler ultrasound combined with serum CA153, CEA and TSGF detection in the diagnosis of breast cancer were 95.15,90.20 and 88.89\% respectively, which were significantly higher than those of any single examination $(\mathrm{P}<0.01$; Table VI). 
Table VI. Comparison of the clinical value of color Doppler ultrasound, CA153, CEA, TSGF single and combined detection in the diagnosis of breast cancer [\% (ratio)].

\begin{tabular}{lccccc}
\hline Detection & Sensitivity & Specificity & Accuracy & Positive predictive value & Negative predictive value \\
\hline Ultrasound & $77.67(80 / 103)$ & $84.00(42 / 50)$ & $79.74(122 / 153)$ & $90.91(80 / 88)$ & $64.62(42 / 65)$ \\
CA153 & $64.08(66 / 103)$ & $92.00(46 / 50)$ & $73.20(112 / 153)$ & $94.29(66 / 70)$ & $55.42(46 / 83)$ \\
CEA & $62.14(64 / 103)$ & $90.00(45 / 50)$ & $71.24(109 / 153)$ & $92.75(64 / 69)$ & $53.57(45 / 84)$ \\
TSGF & $66.02(68 / 103)$ & $90.00(45 / 50)$ & $73.86(113 / 153)$ & $93.15(68 / 73)$ & $56.25(45 / 80)$ \\
Combined detection & $95.15(98 / 103)^{\mathrm{a}}$ & $80.00(40 / 50)$ & $90.20(138 / 153)^{\mathrm{a}}$ & $90.74(98 / 108)$ & $88.89(40 / 45)^{\mathrm{a}}$ \\
$\chi^{2}$ & 39.652 & 1.515 & 20.892 & 1.808 & 18.873 \\
P-value & $<0.001$ & 0.341 & $<0.001$ & 0.771 & 0.001 \\
\hline
\end{tabular}

${ }^{a} \mathrm{P}<0.01$, compared with every single detection. CA153, carbohydrate antigen 153 ; CEA, carcinoembryonic antigen; TSGF, tumor specific growth factor.

\section{Discussion}

Results obtained in a previous study (9) have shown that the early diagnosis of breast cancer can assist early clinical intervention and improve the patients' long-term survival rate and overall quality of life. Considering the number of women affected by breast cancer, to find a simple and effective early screening method for the early diagnosis and treatment of breast cancer is essential.

Ultrasound examination is the first choice for breast disease screening. This method, which is the most widely used breast cancer screening method, is suitable for all age groups and is convenient, non-invasive, non-radioactive, repeatable, and with good patient compliance. In particular, Doppler high-frequency ultrasound has high resolution of details and can clearly display the location, shape, size, boundary, internal structure, echo, calcification and other conditions of the lesion. In addition, Doppler high-frequency ultrasound can clearly visualize the surrounding tissues determining invasion (10). With the increase of probe frequency and the application of blood flow imaging technology, Doppler high-frequency ultrasound can clearly reflect hemodynamic information by detecting tumor angiogenesis and peripheral blood flow characteristics of lesions to further identify benign and malignant breast masses (11), especially cystic and solid masses (12). For dense breast masses, the location and shape can be clearly shown (13). However, ultrasound examination has certain limitations, such as low sensitivity to calcifications and limited ability to accurately identify calcification at the early stages of breast cancer (14). Ultrasound examination has also low sensitivity to the diagnosis of carcinoma-in situ and breast cancer with maximum mass diameter $<1 \mathrm{~cm}$. As the ultrasound image of a small mass is not easy to obtain and there is no obvious typical signs of breast cancer, a small mass can be easily misidentified as a benign lesion. Furthermore, some benign masses are indistinguishable from malignant tumors in terms of image characteristics due to degeneration and ischemia of tissues around the lesions and internal structural disorder of the masses. Moreover, in some cases it has been difficult to confirm some unusual ultrasound images (15). Therefore, there is always a probability of misdiagnosis and missed diagnosis when Doppler ultrasound is used alone. The results of the present study showed that the sensitivity and accuracy of color Doppler ultrasound in the diagnosis of breast cancer were 77.67 and $79.74 \%$, respectively which were not satisfactory.

Tumor markers are molecules secreted by tumor cells or produced by the interaction between tumor and host cells during carcinogenesis. The occurrence or level variation of the tumor markers reflects the existence of a tumor. Such markers can be detected in tissues or body fluids (16). During cell canceration a dramatic increase in the serum levels of these markers is observed, as tumor markers often appear before clinical symptoms. As an in vitro diagnostic method, tumor marker detection is a low cost method with low risk. In addition, tumor marker detection is simple, rapid, quantitative and dynamic and is a commonly used method for the detection of malignant tumors, early diagnosis and prognosis monitoring. However, the results obtained by this method can be affected by various in vitro and in vivo factors, as well as experimental errors. Detection of tumor markers is prone to false positives and false negatives, and therefore the results should be judged with caution.

CA153 is a variant glycoprotein on the surface of breast cells. During malignancy, the activities of salivary enzymes and proteases on the cell membrane are enhanced and the cytoskeleton is destroyed, leading to a fall in cell surface antigen as they are released into the blood stream. The release of CA153 into the blood increases the expression level of this marker in the peripheral blood (17). CA153 is currently used as the most classical tumor marker for screening breast cancer. It has been reported (18) that $80 \%$ of patients with breast cancer metastasis have a high level of serum CA153 and the CA153 level has been reported to be positively correlated with the recurrence and metastasis of breast cancer. Currently, in clinical practice, CA153 is the preferred indicator for monitoring the disease condition. However, there are a few limitations associated with the use of this marker. For example, the level of CA153 in the peripheral blood does not always increase during the early stages of breast cancer ( 0 and I). In addition, a transient increase in CA153 levels (false positive) can be occasionally observed in some benign breast diseases (such as, breast papilloma and breast cysts).

CEA was first found in fetal intestinal and colon cancer tissues. The antigenic determinants of CEA have embryonic characteristics, which explains the name "carcinoembryonic 
antigen'. The level of CEA in healthy individuals is extremely low (in most cases below $3.40 \mathrm{ng} / \mathrm{ml}$ ). When healthy cells transform into cancerous cells, the quantity of CEA secreted by cancer cells rises dramatically. The secreted CEA is diffused in cell membrane, cytoplasm and stroma, although CEA can also be secreted into the cavity edge of cancer gonad tube and its peripheral body fluid (19). Often, CEA levels during the early stages of breast cancer do not show a meaningful rise. These low levels of CEA can be explained by the fact that during the early stages of breast cancer the cells have not yet penetrated the basement membrane and CEA has not been secreted into the peripheral blood. Serum CEA expression levels are of great value in the assessment of breast cancer clinical staging, recurrence and metastasis. Usually, the serum levels of CEA are significantly high during the late stages. In some cases, a transient increase in CEA levels in peripheral blood has been reported in smokers which may lead to false positive results.

TSGF, also known as malignant tumor specific growth factor, is a vascular endothelial growth factor involved in the proliferation of malignant tumors and peripheral capillaries. TSGF is released into the blood stream at the early stages of malignant tumor formation and has no obvious correlation with non-tumor vascular proliferation. TSGF is a powerful indicator in differentiating the cancer cells from the non-cancer cells. As a broad-spectrum tumor marker, TSGF is a valuable marker for the early detection of breast cancer (20). TSGF, certainly, has the merits to be used as an effective screening indicator of breast cancer. After the remission or disappearance of the tumor, the serum levels of TSGF have been reported to decline significantly (21). TSGF can be used for evaluation and prognosis follow ups; however, its sensitivity and specificity are still a matter of debate.

In the present study, color Doppler ultrasound combined with the detection of the serum markers CA153, CEA and TSGF was used in the diagnosis of breast cancer. The results revealed that the combined examinations could complement and confirm each other. Ultrasound can preliminarily distinguish benign and malignant breast tumors based on acoustic characteristics. CA153, CEA and TSGF levels reflect the occurrence and development of breast cancer in terms of molecular biology. The combination of these detection methods makes up for the limitations of each single test. The sensitivity, accuracy and negative predictive value of the combined detection were $95.15,90.20$ and $88.89 \%$, which were significantly higher than those of the single tests, suggesting that the combined detection could reduce misdiagnosis. However, the number of patients included in the present study was relatively small and the study of a bigger sample size will be the aim of our future research.

In conclusion, color Doppler ultrasound combined with the detection of serum markers CA153, CEA and TSGF significantly improved the diagnosis of breast cancer suggesting that the combined detection method could be used as an effective tool that could improve breast cancer diagnosis leading to early diagnosis and clinical intervention.

\section{Acknowledgements}

Not applicable.

\section{Funding}

No funding was received.

\section{Availability of data and materials}

The datasets used and/or analyzed during the present study are available from the corresponding author on reasonable request.

\section{Authors' contributions}

XS conceived the study and wrote the manuscript. BL and CW collected and analyzed the patient general data. SS assisted with the statistical analysis of the data. All authors read and approved the final version of the manuscript.

\section{Ethics approval and consent to participate}

The study was approved by the Ethics Committee of Yantaishan Hospital (Yantai, China). Patients who participated in the study had complete clinical data. Signed written informed consents were obtained from the patients and/or guardians.

\section{Patient consent for publication}

Not applicable.

\section{Competing interests}

The authors declare that they have no competing interests.

\section{References}

1. Ramakrishna N, Temin S, Chandarlapaty S, Crews JR, Davidson NE, Esteva FJ, Giordano SH, Gonzalez-Angulo AM, Kirshner J, Krop I, et al: Recommendations on disease management for patients with advanced human epidermal growth factor receptor 2-positive breast cancer and brain metastases: American society of clinical oncology clinical practice guideline. J Clin Oncol 32: 2100-2108, 2014.

2. Subramanian S and Keating NL: Delays in breast cancer diagnosis after a state policy limiting medicaid enrollment. Cancer 123: 3219-3221, 2017.

3. Tu Z, Xiao R, Xiong J, Tembo KM, Deng X, Xiong M, Liu P Wang $M$ and Zhang Q: CCR9 in cancer: Oncogenic role and therapeutic targeting. J Hematol Oncol 9: 10, 2016.

4. Bidard FC, Hajage D, Bachelot T, Delaloge S, Brain E, Campone M, Cottu P, Beuzeboc P, Rolland E, Mathiot C and Pierga JY: Assessment of circulating tumor cells and serum markers for progression-free survival prediction in metastatic breast cancer: A prospective observational study. Breast Cancer Res 14: R29, 2012.

5. Edge SB and Compton CC: The American joint committee on cancer: The 7th edition of the AJCC cancer staging manual and the future of TNM. Ann Surg Oncol 17: 1471-1474, 2010.

6. Lakhani SR, Ellis IO, Schnitt SJ, Tan PH and van de Vijver MJ (eds): WHO Classification of Tumours of the Breast. IARC Press, Lyon, France, 2012.

7. Kim GR, Choi JS, Han BK, Ko EY, Ko ES and Hahn SY: Combination of shear-wave elastography and color Doppler: Feasible method to avoid unnecessary breast excision of fibroepithelial lesions diagnosed by core needle biopsy. PLoS One 12: e0175380 2017.

8. Lee SH, Chang JM, Cho N, Yi A, Choi SH, Kook SH, Youk JH, Son EJ, Chung J, Cha ES, et al: Abstract P5-02-02: Combined use of elastography and color doppler ultrasound for the evaluation of breast masses detected at screening ultrasound in women with dense breasts. Cancer Res 77: P5, 2017.

9. Printzcancer C: Breast cancer mortality rates decline internationally, with some major exceptions. Cancer 123: 1085, 2017. 
10. Zaleska-Dorobisz U, Kaczorowski K, Pawluś A, Puchalska A and Inglot M: Ultrasound elastography-review of techniques and its clinical applications. Adv Clin Exp Med 23: 645-655, 2014.

11. Sprague BL, Stout NK, Schechter C, van Ravesteyn NT, Cevik M, Alagoz O, Lee CI, van den Broek JJ, Miglioretti DL, Mandelblatt JS, et al: Benefits, harms, and costeffectiveness of supplemental ultrasonography screening for women with dense breasts. Ann Intern Med 162: 157-166, 2015.

12. Chae EY, Moon WK, Kim HH, Kim WH, Cha JH, Shin HJ, Choi WJ, Han W, Noh DY, Lee SB and Ahn SH: Association between ultrasound features and the 21-gene recurrence score assays in patients with oestrogen receptor-positive,HER2-negat ive,invasive breast cancer. PLoS One 11: e0158461, 2016.

13. Costantini M, Belli P, Lombardi R, Franceschini G, Mulè A and Bonomo L: Characterization of solid breast masses: Use of the sonographic breast imaging reporting anddata system lexicon. J Ultrasound Med 25: 649-659, 2006.

14. Bae MS, Seo M, Kim KG, Park IA and Moon WK: Quantitative MRI morphology of invasive breast cancer: Correlation with immunohistochemical biomarkers and subtypes. Acta Radiol 56: 269-275, 2015

15. Ma Y, Li G, Li J and Ren WD: The diagnostic value of superb microvascular imaging (SMI) in detecting blood flow signals of breast lesions: A preliminary study comparing smi to color doppler flow imaging. Medicine (Baltimore) 94: e1502, 2015.

16. Xu MG, Xu JK, Huang HQ and Zhang B: The significance of her 2 gene detection in breast cancer diagnosis and treatment. Mod Oncol 23: 2701-2705, 2015.
17. Bahrami A, Mortazavizadeh MR, Yazdi MF and Chamani M: Serial tumor markers serum carcinoembryonic antigenand cancer antigen 153 assays in detecting symptomatic metastasis in breast cancer patients. East Mediterr Health J 18: 1055-1059, 2012.

18. Duffy MJ, Evoy D and McDermott EW: CA15-3: Uses and limitation as a biomarker for breast cancer. Clin Chim Acta 411: 1869-1874, 2010

19. Svobodova S, Kucera R, Fiala O, Karlikova M, Narsanska A Zedníková I, Treska V, Slouka D, Rousarova M, Topolcan O and Finek J: CEA, CA15-3 and TPS as prognostic factors in the follow-up monitoring of patients after radical surgery for breast cancer. Anticancer Res 38: 465-469, 2018

20. Brooks M: Breast cancer screening and biomarkers. Methods Mol Biol 472: 307-321, 2009.

21. Wang G, Qin Y, Zhang J, Zhao J, Liang Y, Zhang Z, Qin M and Sun Y: Nipple discharge of CA15-3, CA125, CEA and TSGF as a new biomarker panel for breast cancer. Int J Mol Sci 28 9546-9565, 2014. 